\title{
Decoherence in QED at finite temperature
}

\author{
Z. Haba \\ Institute of Theoretical Physics,University of Wroclaw, Wroclaw,Poland \\ e-mail:zhab@ift.uni.wroc.pl
}

\begin{abstract}
We consider a wave packet of a charged particle passing through a cavity filled with photons at temperature $\mathrm{T}$ and investigate its localization and interference properties. It is shown that the wave packet becomes localized and the interference disappears with an exponential speed after a sufficiently long path through the cavity.

PACS numbers:03.65.-w,03.65.Bz,12.20.Ds
\end{abstract}

\section{Introduction}

It has been known for a long time that thermal photons can substantially disturb electron beams in an accelerator [1] [2] [3]. It also has been suggested that the problem of the classical limit of quantum mechanics (at least the problem of decoherence) can be solved in the presence of a reservoir of photons . Some models of an interaction of a quantum system with a reservoir confirming the decoherent behaviour have been discussed in refs. 细 [5][6]. These models are treated as an approximation to the quantum electrodynamics (QED).

We discussed QED and some approximations to QED in ref. [f]. We pointed out a difficulty (resulting from the ultraviolet singularity) with some approximations to QED when applied in a discussion of the decoherence. The singular part of the QED propagator does not contribute to the decoherence in formal semiclassical calculations. In this paper we discuss the regular part. We calculate the time evolution of the density matrix of wave packets in an approximation of a small charge $e$ treating the particle dynamics in a semiclassical approximation. We neglect the vacuum fluctuations considering the effect of thermal photons on the evolution of the density matrix. We show a decoherence effect of the thermal photons. The vacuum fluctuations lead to divergencies at short distances. After a renormalization the contribution of vacuum fluctuations to decoherence is negligible for a large time.

The effect of photons on decoherence has been discussed first by Joos and Zeh [8]. The general arrangement for interference experiments in an environment has been considered in [9]. L. Ford [10] discussed a change of the vacuum 
fluctuations with a change of the environment's geometry (the Casimir effect) and its role in the interference experiments. In such a case the relative strength of the vacuum fluctuations rather than its intrinsic value (which is infinite) is relevant. The decoherent effect of the black body radiation has been studied by Stapp [11] but we disagree with his results and approximation methods.

We are interested in quantum electrodynamics at finite temperature $\mathrm{T}$ determined by the density matrix (the Gibbs state)

$$
\rho=\left(\operatorname{Tr}\left(\exp \left(-\beta H_{R}\right)\right)\right)^{-1} \exp \left(-\beta H_{R}\right)
$$

where $\frac{1}{\beta}=K T, K$ is the Boltzmann constant and $H_{R}$ is the Hamiltonian for the quantum free electromagnetic field.

We compute the correlation functions explicitely

$$
\begin{aligned}
& G_{\beta}\left(0, \mathbf{x} ; t, \mathbf{x}^{\prime}\right)_{j l} \equiv \operatorname{Tr}\left(A_{j}(0, \mathbf{x}) A_{l}\left(t, \mathbf{x}^{\prime}\right) \rho\right)= \\
& \frac{\hbar c}{\pi^{2}} \int d \mathbf{k}|\mathbf{k}|^{-1} \cos \left(\left(\mathbf{x}-\mathbf{x}^{\prime}\right) \mathbf{k}\right) \delta_{j l}^{t r}(\mathbf{k}) \\
& \left(\cos (c|\mathbf{k}| t)\left(\frac{1}{2}+(\exp (\beta \hbar c|\mathbf{k}|)-1)^{-1}\right)-\frac{i}{2} \sin (c|\mathbf{k}| t)\right)
\end{aligned}
$$

where

$$
\delta_{j l}^{t r}(\mathbf{k})=\delta_{j l}-k_{j} k_{l}|\mathbf{k}|^{-2}
$$

The term $\frac{1}{2} \hbar c|\mathbf{k}|$ corresponds to the zero point energy (of vacuum fluctuations) whereas $\hbar c|\mathbf{k}|(\exp (\hbar c|\mathbf{k}| \beta)-1)^{-1}$ is the average energy of thermal photons with the wave number $\mathbf{k}$. The vacuum fluctuation (noise) is a measurable effect and in general cannot be neglected. The virtual photons corresponding to the vacuum fluctuations are not directly observable. Then, the thermal photons are described by the Green's function $G_{t h}=G_{\beta}-G_{\infty}$ (note that $G_{t h}$ is real whereas $G_{\beta}$ and $G_{\infty}$ are complex; in quantum field theory the imaginary part of the Green's function is related to the pair creation and annihilation, so subtracting $G_{\infty}$ means that the processes of pair creation and annihilation are neglected)

$$
G_{t h}\left(\mathbf{x}, \mathbf{x}^{\prime}, t\right)_{j l}=\frac{\hbar c}{2 \pi^{2}} \int d \mathbf{k}|\mathbf{k}|^{-1} \delta_{j l}^{t r}(\mathbf{k}) \cos \left(\left(\mathbf{x}-\mathbf{x}^{\prime}\right) \mathbf{k}\right) \cos (c t|\mathbf{k}|)(\exp (\beta \hbar c|\mathbf{k}|)-1)^{-1}
$$

Let us note that $G_{t h}$ determines the real Gaussian random field. Subsequent computations can be performed either in the Fock space or by means of the functional integration. We do not explain the equivalence of both methods here but refer to our earlier paper [7.

For a small $\left|\mathbf{x}-\mathbf{x}^{\prime}\right|$

$$
G_{t h}\left(\mathbf{x}, \mathbf{x}^{\prime}, t\right)_{j l} \simeq G_{t h}(0,0, t)_{j l}=\delta_{j l} \frac{4}{3} \hbar c \pi^{-1} \int_{0}^{\infty} d k k \cos (c k t)(\exp (\beta \hbar c k)-1)^{-1}
$$

is approximately $\mathbf{x}$-independent. 


\section{The semiclassical approximation}

We approach the semiclassical limit of the wave function in the standard way treating the electromagnetic field $\mathbf{A}$ as a classical field. Then, the quantum electromagnetic field is realized as a random field with the covariance (2) . A solution of the Schrödinger equation

$$
i \hbar \partial_{t} \psi(t, \mathbf{x})=\frac{1}{2 m}\left(-i \hbar \nabla+\frac{e}{c} \mathbf{A}_{t}\right)^{2} \psi(t, \mathbf{x})
$$

with the initial condition $\psi=\exp \left(\frac{i}{\hbar} W\right) \phi$ can be related to the solution of the Hamilton-Jacobi equation $W_{t}$

$$
\partial_{t} W_{t}+\frac{1}{2 m}\left(\nabla W_{t}+\frac{e}{c} \mathbf{A}_{t}\right)^{2}=0
$$

with the initial condition $W_{t=0}(\mathbf{x})=W(\mathbf{x})$. We express $\psi_{t}$ in the form

$$
\psi_{t} \equiv \chi_{t} \phi_{t}=\exp \left(\frac{i}{\hbar} W_{t}\right) \phi_{t}
$$

Then, $\psi_{t}$ is the solution of eq.(4) if and only if $\phi_{t}$ is the solution of the equation

$$
\partial_{t} \phi_{t}=\frac{i \hbar}{2 m} \triangle \phi_{t}-\frac{1}{m}\left(\nabla W_{t}+\frac{e}{c} \mathbf{A}_{t}\right) \nabla \phi_{t}-\frac{1}{2 m}\left(\triangle W_{t}+\frac{e}{c} \operatorname{div} \mathbf{A}_{t}\right) \phi_{t}
$$

with the initial condition $\phi$. In a formal limit $\hbar \rightarrow 0$ the term $\Delta \phi$ can be neglected. In such an approximation the solution of the Schrödinger equation (4) is expressed by the classical flow starting from $\mathbf{x}$ (here $0 \leq s \leq t$ )

$$
\frac{d \mathbf{y}_{s}}{d s}=-\frac{1}{m}\left(\nabla W_{t-s}\left(\mathbf{y}_{s}\right)+\frac{e}{c} \mathbf{A}_{t-s}\left(\mathbf{y}_{s}\right)\right)
$$

Till $o(\hbar)$ terms we have

$$
\psi(t, \mathbf{x})=\exp \left(\frac{i}{\hbar} W_{t}(\mathbf{x})\right) \exp \left(-\int_{0}^{t} \frac{1}{2 m}\left(\triangle W_{t-s}\left(\mathbf{y}_{s}\right)+\frac{e}{c} \operatorname{div} \mathbf{A}_{t-s}\left(\mathbf{y}_{s}\right)\right) d s\right) \phi\left(\mathbf{y}_{t}(\mathbf{x})\right)
$$

If we know the trajectory (e.g., from the Hamilton equations) then we can compute $W_{t}$

$$
W_{t}(\mathbf{x})=W\left(\mathbf{y}_{t}(\mathbf{x})\right)+\int_{0}^{t}\left(\frac{m}{2}\left(\frac{d \mathbf{y}}{d s}\right)^{2}+\frac{e}{c} \mathbf{A}_{s}\left(\mathbf{y}_{s}\right) \frac{d \mathbf{y}}{d s}\right) d s
$$

From the correlation functions (2) of $\mathbf{A}$ it follows that the assumption that $\mathbf{A}(t, \mathbf{x})$ is $\mathbf{x}$-independent is a good approximation for a non-relativistic motion. For a wave packet of momentum $\mathbf{p}$ we have $W=\mathbf{p x}$. Hence, approximately

$$
\frac{d \mathbf{y}_{s}}{d s}=-\frac{1}{m}\left(\mathbf{p}+\frac{e}{c} \mathbf{A}_{t-s}\right)
$$


This is a consistent approximation because for the approximate solution of the Hamilton-Jacobi equation $\nabla W_{s}(\mathbf{x})$ is space independent . Then, for $\mathbf{A}$ which is space independent we have the exact solution of eq.(5)

$$
W_{t}(\mathbf{x})=\mathbf{p x}-\frac{t}{2 m} \mathbf{p}^{2}-\frac{e}{2 m c} \mathbf{p} \int_{0}^{t} \mathbf{A}_{\tau} d \tau-\frac{e^{2}}{2 m c^{2}} \int_{0}^{t} \mathbf{A}_{\tau}^{2} d \tau
$$

As a result of the evolution in an environment of photons (which are not under an observation) the pure state

$$
\psi=\exp (i W / \hbar) \phi
$$

after an average over the states of the quantum electromagnetic field is transformed into a mixed state with the density matrix

$$
\rho_{t}\left(\mathbf{x}, \mathbf{x}^{\prime}\right)=\left\langle\bar{\psi}(\mathbf{x}) \psi\left(\mathbf{x}^{\prime}\right)\right\rangle
$$

where the average is over the electromagnetic field.

We combine the approximation (9)-(10) of a space independent $\mathbf{A}$ with the exact $W_{t}(8)$ in the following approximate expression for $W_{t}$

$$
W_{t}(\mathbf{x})=\mathbf{p x}-\frac{t}{2 m} \mathbf{p}^{2}-\frac{e}{2 m c} \mathbf{p} \int_{0}^{t} \mathbf{A}_{\tau}\left(\mathbf{x}-\frac{\tau}{m} \mathbf{p}\right) d \tau
$$

This expression results from a solution of the equations of motion (7) to the lowest (zeroth) order in $e$. Subsequently, $W_{t}$ in eq.(8) is calculated to the first order in $e$. Inserting $W_{t}$ (12) into eq.(11) we obtain

$$
\begin{aligned}
& \rho_{t}\left(\mathbf{x}, \mathbf{x}^{\prime}\right) \equiv \exp \left(\left(i \mathbf{p} \mathbf{x}^{\prime}-i \mathbf{p x}\right) / \hbar\right) \overline{\phi\left(\mathbf{x}-\frac{t}{m} \mathbf{p}\right)} \phi\left(\mathbf{x}^{\prime}-\frac{t}{m} \mathbf{p}\right) \exp (-S)= \\
& \exp \left(\left(i \mathbf{p} \mathbf{x}^{\prime}-i \mathbf{p x}\right) / \hbar\right) \overline{\phi\left(\mathbf{x}-\frac{t}{m} \mathbf{p}\right)} \phi\left(\mathbf{x}^{\prime}-\frac{t}{m} \mathbf{p}\right) \\
& \exp \left(-\frac{e^{2}}{m^{2} c^{2} \hbar^{2}} \int_{0}^{t} \mathbf{p} G_{t h}((s-\tau) \mathbf{p} / m, s-\tau) \mathbf{p} d s d \tau\right. \\
& +\frac{e^{2}}{2 m^{2} c^{2} \hbar^{2}} \int_{0}^{t} \mathbf{p} G_{t h}\left(\mathbf{x}-\mathbf{x}^{\prime}+(s-\tau) \mathbf{p} / m, s-\tau\right) \mathbf{p} d s d \tau \\
& \left.+\frac{e^{2} \hbar^{2}}{2 m^{2} c^{2} \hbar^{2}} \int_{0}^{t} \mathbf{p} G_{t h}\left(\mathbf{x}^{\prime}-\mathbf{x}+(s-\tau) \mathbf{p} / m, s-\tau\right) \mathbf{p} d s d \tau\right)
\end{aligned}
$$

where $\exp (-S)$ denotes the last factor in eq.(13). If the vacuum fluctuations were to be taken into account then we would need to make the replacement $G_{t h} \rightarrow G_{\beta}=G_{t h}+i \triangle_{F}$, where $\triangle_{F}$ is the Feynman causal propagator (in the notation of Bjorken and Drell [12]).

\section{The estimates of the evolution of the density matrix}

We perform first the integral over time in eq.(13). We denote $\mathbf{y}=\mathbf{x}-\mathbf{x}^{\prime}$, introduce the spherical coordinates $d \mathbf{k}=d k k^{2} d \theta \sin \theta d \phi$ and write $S$ in the 
form

$$
S=\frac{e^{2}}{2 \pi m^{2} c \hbar} \int_{0}^{\infty} d k k \int_{0}^{\pi} d \theta \sin \theta I(\theta, k, p)(\exp (\beta \hbar c k)-1)^{-1}
$$

We restrict ourselves to $|\mathbf{p}| \ll m c$ then

$$
\begin{aligned}
& I(\theta, k, p)=c^{-2} k^{-2}|\mathbf{p}|^{2}\left(1-\cos ^{2} \theta\right) \\
& (2(1-\cos (t c k))-2 \cos \mathbf{k y}+\cos (\mathbf{k y}+c k t)+\cos (\mathbf{k y}-c k t))
\end{aligned}
$$

If $\mathbf{y}$ is large then on the basis of eq.(15) the $\mathbf{y}$-dependent terms are small as a function of $t$ in comparison with other terms, because we have an additional oscillation in $\mathbf{y}$ which makes such a term negligible. The main contribution to $S$ for a large $\mathbf{y}$ is (we write $\alpha=\cos \theta$ )

$$
S=\frac{2 e^{2}}{m^{2} c^{3} \hbar \pi} \mathbf{p}^{2} \int_{-1}^{1} d \alpha\left(1-\alpha^{2}\right) \int_{0}^{\infty} d k k^{-1}(1-\cos (t c k))(\exp (\beta \hbar c k)-1)^{-1}
$$

For a small time $t$ ( and a large $\mathbf{y}$ ) we obtain

$$
\begin{aligned}
& \left|\rho_{t}\right| \approx \exp \left(-\frac{2}{3} \frac{e^{2}}{m^{2} \hbar c \pi} \mathbf{p}^{2} t^{2} \int_{0}^{\infty} d k k(\exp (\beta \hbar c k)-1)^{-1}\right) \\
& \approx \exp \left(-B \frac{e^{2}}{\hbar c}(\mathbf{p} t / m)^{2} l_{d B}^{-2}\right)
\end{aligned}
$$

where $B$ is a constant of order 1 and

$$
l_{d B}=c \hbar \beta
$$

denotes the thermal de Broglie wave length at temperature $\mathrm{T}$. The result (17) means that the decoherence (or simply the effect of the electromagnetic environment) is visible after a particle makes a path comparable with de Broglie wave length.

Let us consider now a large $t \geq 0$ in eq.(16). We apply the formula

$$
1-\cos w=w \int_{0}^{1} d \gamma \sin (\gamma w)
$$

and the formula 3.911 of Gradshtein and Ryzhik [14]

$$
\int_{0}^{\infty} d u \sin (a u)(\exp (\beta u)-1)^{-1}=\frac{\pi}{2 \beta} \operatorname{coth}\left(\frac{\pi a}{\beta}\right)-\frac{1}{2 a}
$$

Then, we obtain

$$
\begin{aligned}
& S=\frac{4}{3} \frac{t e^{2}}{m^{2} c^{2} \hbar \pi} \mathbf{p}^{2} \int_{0}^{1} d \gamma \int_{0}^{\infty} d k \sin (t c k \gamma)(\exp (\beta \hbar c k)-1)^{-1} \\
& =\frac{4}{3} \frac{e^{2}}{m^{2} c^{3} \hbar \pi} \mathbf{p}^{2} \int_{0}^{t} d \gamma\left(\frac{\pi}{\beta \hbar} \operatorname{coth}\left(\frac{\pi \gamma}{\beta \hbar}\right)-\frac{1}{\gamma}\right) \\
& =\frac{4}{3} \frac{e^{2}}{m^{2} c^{3} \hbar \pi} \mathbf{p}^{2} \ln \left(\frac{\beta \hbar}{\pi t} \sinh \left(\frac{\pi t}{\beta \hbar}\right)\right) \approx \frac{\left.\mathbf{p}\right|^{2}}{m^{2} c^{2}} \frac{e^{2}}{c \hbar} \frac{c t}{l d B}
\end{aligned}
$$


for a large $t$ (and a large $|\mathbf{y}|$ ).

Let us consider next small $t$ together with a small $\mathbf{y}$. Then, we can set $s=\tau=0$ in the argument of $G_{t h}$ in eq.(13). In such a case

$$
\begin{aligned}
& S \simeq \frac{e^{2}}{m^{2} c_{\pi}} \mathbf{p}^{2} t^{2} \int_{0}^{\infty} d k k \int_{0}^{\pi} d \theta \sin \theta \\
& \left(1-\cos ^{2} \theta\right)(1-\cos (k \cos \theta|\mathbf{y}|))(\exp (\beta \hbar c k)-1)^{-1}
\end{aligned}
$$

The formula (19) is relevant only for a small $\mathbf{y}$ (for a large $\mathbf{y}$ we return to eq.(17)). From eq.(19) for a small $\mathbf{y}$ and small $t$ we obtain

$$
\begin{aligned}
& \rho_{t}\left(\mathbf{x}, \mathbf{x}^{\prime}\right) \approx \exp \left(-\frac{2 e^{2}}{15 \pi m^{2} \hbar c} \mathbf{p}^{2} t^{2} \mathbf{y}^{2} \int_{0}^{\infty} d k k^{3}(\exp (\hbar c \beta k)-1)^{-1}\right) \\
& \approx \exp \left(-B \frac{e^{2}}{\hbar c}\left(\frac{\mathbf{p} t}{m}\right)^{2} \mathbf{y}^{2} l_{d B}^{-4}\right)
\end{aligned}
$$

where $B$ is a constant of order 1 . Again we can conclude that the decoherence is visible on distances comparable to the thermal de Broglie length.

For a large $t$ we neglect the quickly oscillating $t$-dependent terms in eq.(15). Then, similarly as in the computations in eq.(18) (with $\alpha=\cos \theta$ )

$$
\begin{aligned}
& S=\frac{e^{2}}{\pi m^{2} c^{3} \hbar} \mathbf{p}^{2}|\mathbf{y}| \int_{0}^{1} d \gamma \int_{-1}^{1} d \alpha \alpha\left(1-\alpha^{2}\right) \int_{0}^{\infty} d k(\exp (\beta \hbar c k)-1)^{-1} \sin (k \gamma \alpha|\mathbf{y}|) \\
& =\frac{2 e^{2}}{\pi m^{2} c^{3} \hbar} \mathbf{p}^{2} \int_{0}^{1} d \alpha\left(1-\alpha^{2}\right) \ln \left(\frac{\beta \hbar c}{\pi \alpha|\mathbf{y}|} \sinh \left(\frac{\pi \alpha|\mathbf{y}|}{\beta \hbar c}\right)\right) \approx \frac{e^{2}}{\hbar c} \frac{\mathbf{p}^{2}}{m^{2} c^{2}} \frac{|\mathbf{y}|}{l_{d B}}
\end{aligned}
$$

for a large $\mathbf{y}$ (and a large $t$ ).

For a small $|\mathbf{y}|$ (and a large $t$ ) the $t$-dependent terms in eq.(15) can be neglected. Then, the integral (14) gives

$$
S=\frac{2}{15} \frac{e^{2}}{\pi m^{2} c^{3} \hbar}|\mathbf{p}|^{2}|\mathbf{y}|^{2} l_{d B}^{-2} \int_{0}^{\infty} d k k(\exp k-1)^{-1}
$$

Hence,

$$
\left|\rho_{t}\right| \approx \exp \left(-B \frac{e^{2}}{\hbar c}|\mathbf{p}|^{2}(m c)^{-2}|\mathbf{y}|^{2} l_{d B}^{-2}\right)
$$

At this point it is useful to recall the definition of the Wigner function

$$
\mathcal{W}(\mathbf{q}, \mathbf{k})=(2 \pi \hbar)^{-3} \int d \mathbf{u} \exp (i \mathbf{k u} / \hbar) \rho(\mathbf{q}+\mathbf{u} / 2, \mathbf{q}-\mathbf{u} / 2)
$$

If $\rho \approx \exp \left(-\frac{a}{2} \mathbf{y}^{2}-i \mathbf{p y} / \hbar\right)$ then

$$
\mathcal{W}(\mathbf{q}, \mathbf{k}) \simeq \exp \left(-\frac{1}{2 a \hbar^{2}}(\mathbf{p}-\mathbf{k})^{2}\right) w(\mathbf{q}, \mathbf{k})
$$

Such a behaviour means a localization on the classical momentum p. If (as in eq.(18) for a large time) $\rho_{t}=\exp (-i \mathbf{p y}-S) \simeq \exp \left(-i \mathbf{p y}-b \mathbf{p}^{2} t\right)$ then approximately

$$
\partial_{t} \rho_{t} \approx-b\left[\mathbf{P},\left[\mathbf{P}, \rho_{t}\right]\right]
$$


where $\mathbf{P}=-i \hbar \nabla$ is the quantum momentum operator. Then

$$
\partial_{t} \mathcal{W}_{t}(\mathbf{q}, \mathbf{k}) \approx-b \mathbf{k}^{2} \mathcal{W}_{t}(\mathbf{q}, \mathbf{k})
$$

For N-particles with momenta $\mathbf{p}_{j}$ we consider the initial state of the form

$$
\psi=\exp \left(\frac{i}{\hbar} \sum_{j=1}^{N} \mathbf{p}_{j} \mathbf{x}_{j}\right) \phi
$$

Then, $W_{t}$ in eq.(12) is a sum of the Hamilton-Jacobi functions for each particle. The subsequent expectation value over the electromagnetic field gives an exponential with a sum of pairings between the $N$ particles. Hence, it can be expected that $\rho_{t}$ behaves as $\exp \left(-R N^{2} t^{2}\right)$ for a small $\mathrm{t}$ and as $\exp \left(-R N^{2}|t|\right)$ for a large $t$ with a certain constant $R$ (note that the behaviour $\exp (-R N|t|$ ) for another model has been obtained by Unruh [13]). Such a result is valid for $\mathrm{N}$ particles of equal charges under the assumption that the terms in the exponential of the form (13) have equal signs. The contributions add if the momenta have a distinguished direction. If the directions are random then the contributions from different particles cancel one another. A similar cancellation takes place if the system is neutral, i.e., if the charges $e_{j}$ can have different signs. Then, in the sum in an exponential of the form (13) we shall have $e_{j} e_{k} \mathbf{p}_{j} \mathbf{p}_{k}$ multiplying $G_{t h}$. Hence, there can be cancellations from different charges $e_{j}$ even if there is a distinguished direction of the momenta. Under an assumption that the contributions in the exponential do not cancel one another we obtain for a large distance between any pair of coordinates and a large $t$

$$
\left|\rho_{t}\right| \approx \exp \left(-B \frac{e^{2}}{c \hbar} N^{2}\left(\frac{|\mathbf{p}|}{m c}\right)^{2} \frac{c t}{l_{d B}}\right)
$$

where B is a constant of order 1 . Such a decay rate means that the decay is visible if the distance achieved by a particle (with the mean momentum $\mathbf{p}$ ) is comparable with the de Broglie wave length. The time needed for this purpose can be short if $N$ is large

$$
\frac{e^{2}}{c \hbar} N^{2}>\left(\frac{m c}{|\mathbf{p}|}\right)^{2} \frac{l_{d B}}{c t}
$$

\section{Disappearance of the interference}

So far we have discussed the decay of $\rho_{t}\left(\mathbf{x}, \mathbf{x}^{\prime}\right)$ for varying $t$ and $\mathbf{y}=\mathbf{x}-\mathbf{x}^{\prime}$. The disappearance of the off-diagonal matrix elements indicates a classical behaviour of quantum probabilities in the state $\rho_{t}$. We investigate next what happens with the interference describing a typical quantum phenomenon. We consider a superposition of two wave packets

$$
\psi(\mathbf{x})=\exp \left(i \mathbf{p}^{(1)} \mathbf{x} / \hbar\right) \phi(\mathbf{x})+\exp \left(i \mathbf{p}^{(2)} \mathbf{x} / \hbar\right) \phi(\mathbf{x})
$$


Then, at the point $\mathbf{x}$ (on the screen) after an evolution through a cavity filled with thermal photons the probability density $\left\langle\left|\psi_{t}(\mathbf{x})\right|^{2}\right\rangle$ is equal to the diagonal part of the density matrix

$$
\rho_{t}=\left\langle\mid \psi_{t}\right\rangle\left\langle\psi_{t} \mid\right\rangle
$$

For each packet we have the Hamilton-Jacobi function

$$
W_{t}(\mathbf{x})=\mathbf{p x}-t \frac{\mathbf{p}^{2}}{2 m}-\frac{\mathbf{p} e}{m c} \int_{0}^{t} \mathbf{A}\left(\mathbf{y}_{s}(\mathbf{x}), s\right) d s
$$

Under the time evolution

$$
\psi \rightarrow \psi_{t}=\exp \left(i W_{t}^{(1)} / \hbar\right) \phi_{t}^{(1)}+\exp \left(i W_{t}^{(2)} / \hbar\right) \phi_{t}^{(2)}
$$

In our semiclassical approximation $\left.\phi(\mathbf{x}) \rightarrow \phi\left(\mathbf{y}_{t}(\mathbf{x})\right)\right)$. Then, for weak fields we neglect the dependence of the paths on the electromagnetic field, i.e., we consider straight lines

$$
\mathbf{y}_{s}^{(1)}=\mathbf{x}-\frac{s}{m} \mathbf{p}^{(1)}
$$

and

$$
\mathbf{y}_{s}^{(2)}=\mathbf{x}-\frac{s}{m} \mathbf{p}^{(2)}
$$

In this approximation the expectation value (23) is

$$
\begin{aligned}
& \left\langle\left|\psi_{t}(\mathbf{x})\right|^{2}\right\rangle=\left|\phi\left(\mathbf{x}-\frac{t}{m} \mathbf{p}^{(1)}\right)\right|^{2}+\left|\phi\left(\mathbf{x}-\frac{t}{m} \mathbf{p}^{(2)}\right)\right|^{2}+ \\
& \left(\overline{\phi\left(\mathbf{x}-\frac{t}{m} \mathbf{p}^{(2)}\right)} \phi\left(\mathbf{x}-\frac{t}{m} \mathbf{p}^{(1)}\right)\right. \\
& \exp \left(-\frac{i}{\hbar}\left(\mathbf{p}^{(2)}-\mathbf{p}^{(1)}\right) \mathbf{x}+\frac{i t}{2 m \hbar}\left(\left(\mathbf{p}^{(2)}\right)^{2}-\left(\mathbf{p}^{(1)}\right)^{2}\right)\right)+ \\
& \left.\exp \left(\frac{i}{\hbar}\left(\mathbf{p}^{(2)}-\mathbf{p}^{(1)}\right) \mathbf{x}-\frac{i t}{2 m \hbar}\left(\left(\mathbf{p}^{(2)}\right)^{2}-\left(\mathbf{p}^{(1)}\right)^{2}\right)\right) \overline{\phi\left(\mathbf{x}-\frac{t}{m} \mathbf{p}^{(1)}\right)} \phi\left(\mathbf{x}-\frac{t}{m} \mathbf{p}^{(2)}\right)\right) \\
& \exp \left(\frac{e^{2}}{2 m^{2} c^{2} \hbar^{2}} \int_{0}^{t} \mathbf{p}^{(1)} G_{t h}\left(\frac{s}{m} \mathbf{p}^{(1)}-\frac{\tau}{m} \mathbf{p}^{(2)}, s-\tau\right) \mathbf{p}^{(2)} d s d \tau\right. \\
& +\frac{e^{2}}{2 m^{2} c^{2} \hbar^{2}} \int_{0}^{t} \mathbf{p}^{(1)} G_{t h}\left(\frac{s}{m} \mathbf{p}^{(2)}-\frac{\tau}{m} \mathbf{p}^{(1)}, s-\tau\right) \mathbf{p}^{(2)} d s d \tau \\
& -\frac{e^{2}}{2 m^{2} c^{2} \hbar^{2}} \int_{0}^{t} \mathbf{p}^{(1)} G_{t h}\left(\frac{s}{m} \mathbf{p}^{(1)}-\frac{\tau}{m} \mathbf{p}^{(1)}, s-\tau\right) \mathbf{p}^{(1)} d s d \tau \\
& \left.-\frac{e^{2}}{2 m^{2} c^{2} \hbar^{2}} \int_{0}^{t} \mathbf{p}^{(2)} G_{t h}\left(\frac{s}{m} \mathbf{p}^{(2)}-\frac{\tau}{m} \mathbf{p}^{(2)}, s-\tau\right) \mathbf{p}^{(2)} d s d \tau\right) \equiv \rho_{t}^{(1)}+\rho_{t}^{(2)}+\rho_{t}^{(12)}
\end{aligned}
$$

Without detailed calculations we can obtain the behaviour for a small t. Let $s=\tau=0$ in $G_{t h}$ in eq.(25) then from eq.(3) $G_{t h}(0,0) \simeq B \delta_{j l} \beta^{-2} \hbar^{-1} c^{-1}$. It follows that

$$
\left|\rho_{t}^{(12)}\right| \simeq \exp \left(-B \frac{e^{2}}{\hbar c} l_{d B}^{-2}\left(t\left|\mathbf{p}^{(2)}-\mathbf{p}^{(1)}\right| / m\right)^{2}\right)
$$

Hence, if the decoherence is to be visible the distance between the particles after time $t$ must be of the order of the de Broglie length. The calculations for a large time are more involved. Let us denote

$$
\left|\rho_{t}^{(12)}\right| \equiv \exp \left(-S_{12}\right)
$$


We obtain for $S_{12}$

$$
\begin{aligned}
& S_{12}=\frac{2}{3} \frac{e^{2}}{\pi m^{2} c^{3} \hbar}\left|\mathbf{p}^{(2)}-\mathbf{p}^{(1)}\right|^{2} \int_{0}^{\infty} \frac{d k}{k}(\exp (\beta \hbar c k)-1)^{-1}(1-\cos (t c k)) \\
& =\frac{2}{3} \frac{e^{2}}{\pi m^{2} c^{3} \hbar}\left|\mathbf{p}^{(2)}-\mathbf{p}^{(1)}\right|^{2} c t \int_{0}^{1} d \gamma \int_{0}^{\infty} d k(\exp (\beta \hbar c k)-1)^{-1} \sin (\gamma c k t) \\
& =\frac{2}{3} \frac{e^{2}}{\pi m^{2} c^{2} \hbar}\left|\mathbf{p}^{(2)}-\mathbf{p}^{(1)}\right|^{2} \int_{0}^{1} d \gamma\left(\frac{t \pi}{2 \beta \hbar c} \operatorname{coth}\left(\frac{\pi \gamma t}{\beta \hbar}\right)-\frac{1}{2 \gamma c}\right) \\
& =\frac{1}{3} \frac{e^{2}}{\pi m^{2} c^{3} \hbar}\left|\mathbf{p}^{(2)}-\mathbf{p}^{(1)}\right|^{2} \ln \left(\frac{\beta \hbar}{\pi t} \sinh \left(\frac{t \pi}{\beta \hbar}\right)\right) \\
& \approx \frac{e^{2}}{\hbar c} \frac{c t}{l_{d B}}\left|\mathbf{p}^{(2)}-\mathbf{p}^{(1)}\right|^{2} m^{-2} c^{-2}
\end{aligned}
$$

for a large t. Hence, the mixed term in eq. (25) is multiplied by $\exp \left(-S_{12}\right)$ which decays as $\exp (-R t)$. Such a behaviour of the probability density proves that the thermal photons lead to the classical addition of probabilities instead of the quantum addition of amplitudes showing the decoherence phenomenon in a physical model of an electron-photon interaction.

\section{Conclusions}

We have discussed a model of a quantum charged particle interacting with a quantum electromagnetic field at finite temperature. We have calculated the time evolution of the density matrix in a semiclassical approximation for the wave function and in a weak coupling approximation for the particle-photon interaction. In contradistinction to refs. [4] 河] [6] we do not apply the approximation of a linear coordinate coupling to the environment. For a small time and small space separations we obtain an exponential in time and space decay $\rho_{t} \approx \exp \left(-b t^{2}\left|\mathbf{x}-\mathbf{x}^{\prime}\right|^{2}\right)$ of off-diagonal matrix elements (decoherence). For a large time the decay achieves its stationary value (18) and (21). The time and space scale is determined by the thermal de Broglie wave length. If we have a large number $N$ of charged particles then the decoherence rate can increase as $N^{2}$. The density matrix elements decay as $\rho_{t} \approx \exp (-b t)$ for a large time. Such a behaviour is in agreement with the Lindblad dynamics if the dissipative part of the dynamics has the form (22). Such Lindblad dynamics has been discussed in refs. 15] 116]. The Lindblad dynamics resulting from a linear coordinate coupling to the environment (studied in 4 45]) is of a different type. It could be described by a replacement of the momentum operator by a position operator in eq.(22) (a coupling to the environment linear in the momentum as well as in the coordinate has been discussed by Leggett in ref.[17]). We have studied the interference as another typical aspect of a quantum behaviour. We have shown that in an environment of photons the interference disappears with an exponential speed (eqs.(26)-(27)). Our results suggest an arrangement for an experiment. Such experiments could verify the QED beyond the usual perturbative approximation as well as the principle of the wave function reduction in a non-selective measurement. 


\section{References}

[1] G.R. Blumenthal and R.J. Gould, Rev.Mod.Phys.42,237(1970)

[2] L.S. Brown and R. S. Steinke, Am.J.Phys.65,304(1997)

[3] B. Dehning et al, Phys.Lett.B249,145(1990)

[4] A.O. Caldeira and A.J. Leggett, Physica A121,587(1983)

[5] W.G. Unruh and W.H.Zurek, Phys.Rev.D40,1071(1989)

[6] P.M.V.B. Barone and A.O.Caldeira,Phys.Rev.A43,57(1991)

[7] Z. Haba, Journ.Math.Phys.39,1766(1998)

[8] E. Joos and H.D. Zeh, Z. Phys. B59,223(1985)

[9] A. Stern, Y. Aharonov and Y. Imry, Phys.Rev. A41,3436(1990)

[10] L.H. Ford, Phys.Rev. D47,5571(1993)

[11] H.P. Stapp, Phys.Rev.A46,6860(1992)

[12] J.D. Bjorken and S.D. Drell, Relativistic Quantum Fields, McGrawHill,1965

[13] W.G. Unruh, Phys.Rev.A51,992(1995)

[14] I.S. Gradshtein and I.M. Ryzhik, Tables of Integrals,Sums, Series and Products, Nauka,Moscow,1971 (in Russian)

[15] A. Sandulescu and H. Scutaru, Ann.Phys.(N.Y.) 173,277(1987)

[16] Z. Haba, Phys.Rev.A57,4034(1998)

[17] A.J. Leggett, Phys.Rev.B30,1208(1984) 